\title{
Flavour decomposition of electromagnetic transition form factors of nucleon resonances
}

\author{
Jorge Segovia ${ }^{1, a}$ and Craig D. Roberts ${ }^{2}$ \\ ${ }^{1}$ Physik-Department, Technische Universität München, James-Franck-Str. 1, D-85748 Garching, Germany \\ ${ }^{2}$ Physics Division, Argonne National Laboratory, Argonne, Illinois 60439, USA
}

\begin{abstract}
In Poincaré-covariant continuum treatments of the three valence-quark boundstate problem, the force behind dynamical chiral symmetry breaking also generates nonpointlike, interacting diquark correlations in the nucleon and its resonances. We detail the impact of these correlations on the nucleon's elastic and nucleon-to-Roper transition electromagnetic form factors, providing flavour-separation versions that can be tested at modern facilities.
\end{abstract}

\section{Introduction}

Dynamical chiral symmetry breaking (DCSB) is a theoretically-established feature of Quantum Chromodynamics (QCD) and the most important mass generating mechanism for visible matter in the Universe, being responsible for approximately $98 \%$ of the proton's mass. A fundamental expression of DCSB is the behaviour of the quark mass-function, $M(p)$. This appears in the dressed-quark propagator which may be obtained as a solution to the most famous and simple QCD's Dyson-Schwinger equation: the gap equation [1]. The nontrivial character of the mass function arises primarily because a dense cloud of gluons comes to clothe a low-momentum quark. It explains how an almost-massless parton-like quark at high energies transforms, at low energies, into a constituent-like quark with an effective mass of around $350 \mathrm{MeV}$.

DCSB ensures the existence of nearly-massless pseudo-Goldstone modes (pions), but another equally important consequence of DCSB is less well known. Namely, any interaction capable of creating pseudo-Goldstone modes as bound-states of a light dressed-quark and -antiquark, and reproducing the measured value of their leptonic decay constants, will necessarily also generate strong colour-antitriplet correlations between any two dressed quarks contained within a baryon. Although a rigorous proof within QCD cannot be claimed, this assertion is based upon an accumulated body of evidence, gathered in two decades of studying two- and three-body bound-state problems in hadron physics (the interested reader is referred to the discussion in Ref. [2] and to Refs. [21-35] cited therein). No realistic counter examples are known; and the existence of such diquark correlations is also supported by simulations of lattice-regularised QCD [3, 4].

The diquark correlations predicted to exist within baryons are not the static, pointlike degrees-offreedom which were historically introduced in order to solve the fact that many states predicted within

ae-mail: jorge.segovia@tum.de 

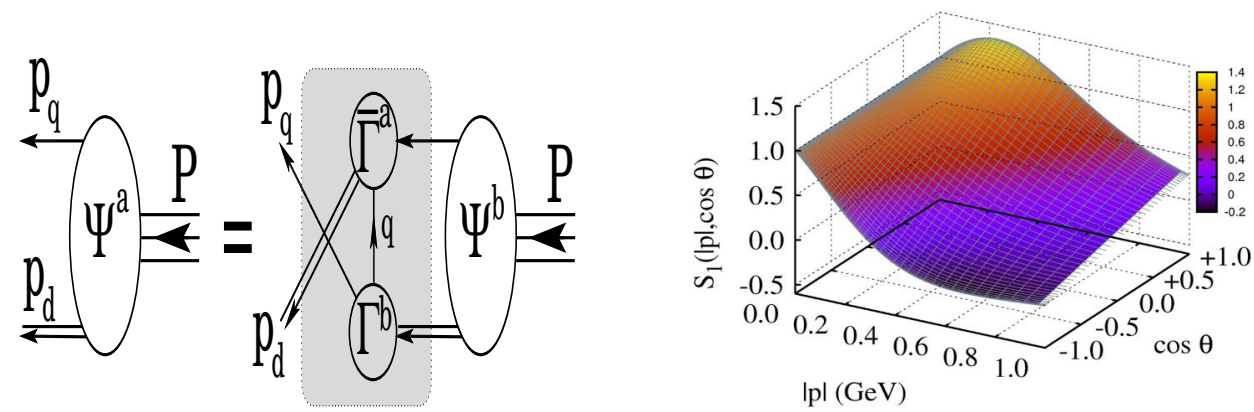

Figure 1. Left panel: Poincaré covariant Faddeev equation. $\Psi$ is the Faddeev amplitude for a baryon of total momentum $P=p_{q}+p_{d}$, where $p_{q, d}$ are, respectively, the momenta of the quark and diquark within the bound-state. The shaded area demarcates the Faddeev equation kernel: single line, dressed-quark propagator; $\Gamma$, diquark correlation amplitude; and double line, diquark propagator. Right panel: Dominant piece in the nucleon's eight-component Poincaré-covariant Faddeev amplitude: $S_{1}(|p|, \cos \theta)$. In the nucleon rest frame, this term describes that piece of the quark-scalar-diquark relative momentum correlation which possesses zero intrinsic quark-diquark orbital angular momentum, i.e. $L=0$, before the propagator lines are reattached to form the Faddeev wave function. Referring to the right panel of Fig. $1, p=P / 3-p_{q}$ and $\cos \theta=p \cdot P / \sqrt{p^{2} P^{2}}$. The amplitude is normalised such that its $U_{0}$ Chebyshev moment is unity at $|p|=0$, see Ref. [10].

the $S U(6) \otimes O(3)$ quark model were missing from the observed spectrum. The modern dynamical diquark correlations are nonpointlike and fully interacting, and each of a baryon's three dressedquarks is involved in every type of correlation to the fullest extent allowed by its quantum numbers and those of the bound state. One should therefore expect the spectrum obtained in the presence of such correlations to be as rich as that allowed by a three-constituent quark model $[5,6]$.

It has been argued that the presence of diquark correlations should have observable consequences on the elastic and transition form factors of nucleon resonances [2,7]. Many related insights have been revealed in a series of recent articles [8-11] focused on the calculation of the Nucleon, Delta and Roper elastic and transition form factors using a widely-used leading-order (rainbow-ladder) truncation of QCD's Dyson-Schwinger equations and comparing results between a QCD-based framework and a vector $\otimes$ vector contact interaction. It is our purpose here reviewing some of the outcomes of such studies, paying particular attention to those directly related with strong diquark correlations and can be tested at modern facilities.

\section{Baryon structure and the nucleon's form factors}

In the quark+diquark picture, baryons are described by the Poincaré covariant Faddeev equation depicted in the left panel of Fig. 1. Two main contributions appear in the binding energy: i) the formation of tight diquark correlations and ii) the quark exchange depicted in the shaded area of the left panel of Fig. $1^{1}$. This exchange ensures that diquark correlations within the baryon are fully dynamical: no quark holds a special place because each one participates in all diquarks.

The quark+diquark structure of the nucleon is elucidated in the right panel of Fig. 1, which depicts the leading component of its Faddeev amplitude: $S_{1}(|p|, \cos \theta)$ [10]. This function describes a piece of the quark+scalar-diquark relative momentum correlation. Notably, in this solution of a realistic

\footnotetext{
${ }^{1}$ Whilst an explicit three-body term might affect fine details of baryon structure, the dominant effect of non-Abelian multigluon vertices is expressed in the formation of diquark correlations [12].
} 

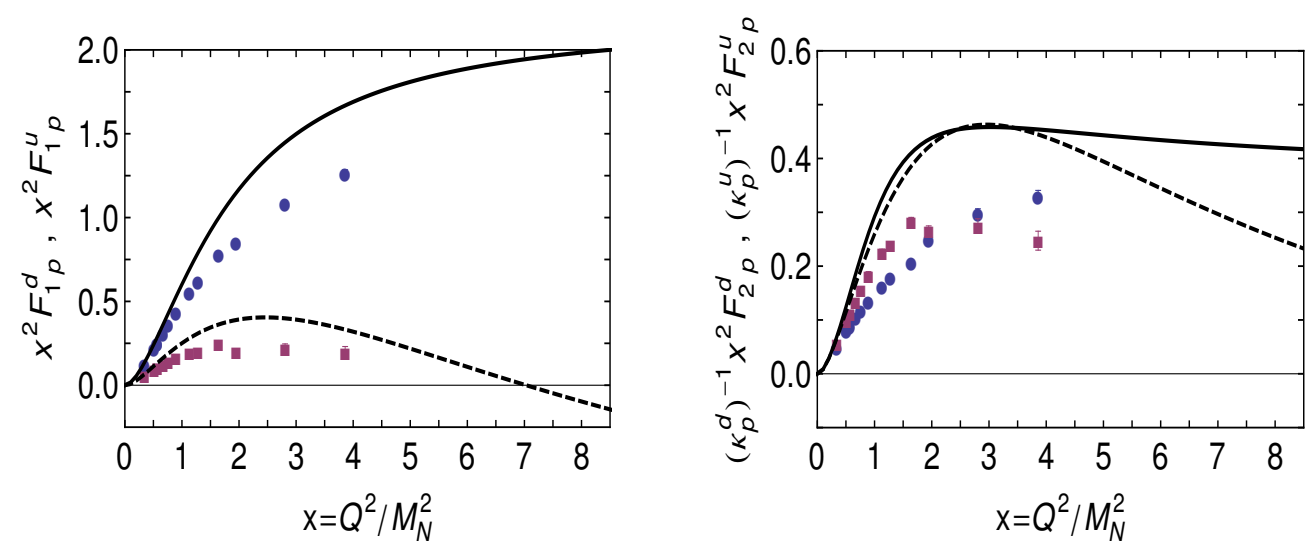

Figure 2. Left panel: Flavour separation of the proton's Dirac form factor as a function of $x=Q^{2} / M_{N}^{2}$. The results have been obtained using a framework built upon a Faddeev equation kernel and interaction vertices that possess QCD-like momentum dependence. The solid-curve is the $u$-quark contribution, and the dashed-curve is the $d$-quark contribution. Experimental data taken from Ref. [15] and references therein: circles $-u$-quark; and squares $-d$-quark. Right panel: Same for Pauli form factor. We define $\kappa_{u}=F_{2 p}^{u}(0)$ and $\kappa_{d}=F_{2 p}^{d}(0)$ and they can be expressed in terms of the proton $\left(\kappa_{p}\right)$ and neutron $\left(\kappa_{n}\right)$ anomalous magnetic moments $[2,15]$.

Faddeev equation there is strong variation with respect to both arguments. Support is concentrated in the forward direction, $\cos \theta>0$, so that alignment of $p$ and $P$ is favoured; and the amplitude peaks at $\left(|p| \simeq m_{N} / 6, \cos \theta=1\right)$, whereat $p_{q} \sim p_{d} \sim P / 2$ and hence the natural relative momentum is zero. In the antiparallel direction, $\cos \theta<0$, support is concentrated at $|p|=0$, i.e. $p_{q} \sim P / 3, p_{d} \sim 2 P / 3$. A realistic nucleon amplitude is evidently a complicated function; and significant structure is lost if simple interactions and/or truncations are employed in building the Faddeev kernel, e.g. extant treatments of a momentum-independent quark-quark interaction - a contact interaction - produce a Faddeev amplitude that is also momentum independent $[13,14]$, a result exposed as unrealistic by the right panel of Fig. 1 for any probe sensitive to the nucleon interior.

The presence of diquark correlations inside the nucleon (and its resonances) must be evident in numerous empirical differences between the response of the bound-state's doubly- and singlyrepresented quarks to any probe whose wavelength is small enough to expose the diquarks' nonpointlike character. In connection with electromagnetic probes, Fig. 2 displays the proton's flavour separated Dirac and Pauli form factors. The salient features of the data are: the $d$-quark contribution to $F_{1}^{p}$ is far smaller than the $u$-quark contribution; $F_{2}^{d} / \kappa_{d}>F_{2}^{u} / \kappa_{u}$ on $x<2$ but this ordering is reversed on $x>2$; and in both cases the $d$-quark contribution falls dramatically on $x>3$ whereas the $u$-quark contribution remains roughly constant.

It is natural to seek an explanation for the pattern of behaviour in Fig. 2. The proton contains scalar and pseudovector diquark correlations. The dominant piece of its Faddeev wave function is $u[u d]$; namely, a $u$-quark in tandem with a $[u d]$ scalar correlation, which produces $62 \%$ of the proton's normalisation. If this were the sole component, then photon- $d$-quark interactions within the proton would receive a $1 / x$ suppression on $x>1$, because the $d$-quark is sequestered in a soft correlation, whereas a spectator $u$-quark is always available to participate in a hard interaction. At large $x=$ $Q^{2} / m_{N}^{2}$, therefore, scalar diquark dominance leads one to expect $F^{d} \sim F^{u} / x$. Available data are consistent with this prediction but measurements at $x>4$ are necessary for confirmation. 

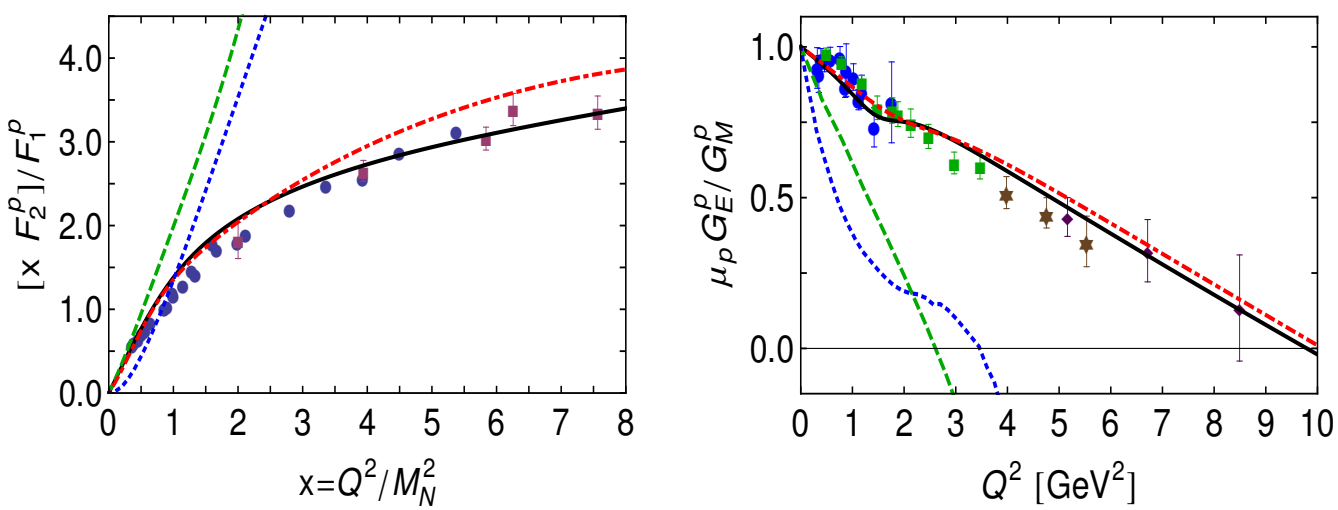

Figure 3. Left panel: Proton ratio $R_{21}(x)=x F_{2}(x) / F_{1}(x), x=Q^{2} / M_{N}^{2}$. Curves: solid (black) - full result, determined from the complete proton Faddeev wave function and current; dot-dashed (red) - momentum-dependence of scalar-diquark contribution; dashed (green) - momentum-dependence produced by that piece of the scalar diquark contribution to the proton's Faddeev wave function which is purely $S$-wave in the rest-frame; dotted (blue) - momentum-dependence of pseudovector diquark contribution. All partial contributions have been renormalised to produce unity at $x=0$. Experimental data taken from Ref. [15]. Right panel: Computed ratio of proton electric and magnetic Sachs form factors. The legend for the curves is the same than that of the lower-left panel. Data: circles (blue) [16]; squares (green) [17]; asterisks (brown) [18]; and diamonds (purple) [19].

Consider now the ratio $R_{21}(x)=x F_{2}(x) / F_{1}(x)$ of the proton. A clear conclusion from the left panel of Fig. 3 is that pseudovector diquark correlations have little influence on the momentum dependence of $R_{21}(x)$. Their contribution is indicated by the dotted (blue) curve, which was obtained by setting the scalar diquark component of the proton's Faddeev amplitude to zero and renormalising the result to unity at $x=0$. As apparent from the dot-dashed (red) curve, the evolution of $R_{21}(x)$ with $x$ is primarily determined by the proton's scalar diquark component. As we have explained above, in this component, the valence $d$-quark is sequestered inside the soft scalar diquark correlation so that the only objects within the nucleon which can participate in a hard scattering event are the valence $u$-quarks. The scattering from the proton's valence $u$-quarks is responsible for the momentum dependence of $R_{21}(x)$. However, the dashed (green) curve in the left panel of Fig. 3 reveals something more, i.e. components of the nucleon associated with quark-diquark orbital angular momentum $L \geq 1$ in the nucleon rest frame are critical in explaining the data. Notably, the presence of such components is an inescapable consequence of the self-consistent solution of a realistic Poincaré-covariant Faddeev equation for the nucleon.

It is natural now to consider the ratio of the proton electric and magnetic Sachs form factors: $R_{E M}\left(Q^{2}\right)=\mu_{p} G_{E}\left(Q^{2}\right) / G_{M}\left(Q^{2}\right), \mu_{p}=G_{M}(0)$, drawn in the right panel of Fig. 3. As with $R_{21}$, the momentum dependence of $R_{E M}\left(Q^{2}\right)$ is principally determined by the scalar diquark component of the proton. Moreover, the rest-frame $L \geq 1$ terms are again seen to be critical in explaining the data: the behaviour of the dashed (green) curve highlights the impact of omitting these components.

\section{The $\gamma^{*} N \rightarrow N(1440)$ Transition}

Jefferson Lab experiments [20-22, 24] have yielded precise nucleon-to-Roper transition form factors and thereby exposed the first zero seen in any hadron form factor or transition amplitude. We have 

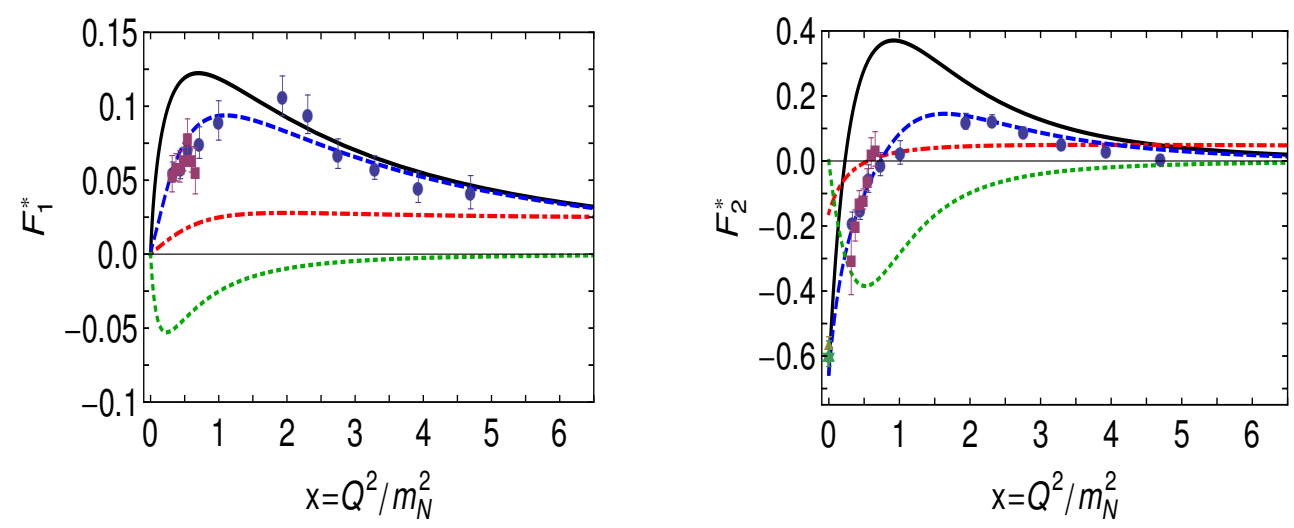

Figure 4. Left panel - Dirac transition form factor $F_{1}^{*}(x)$ as a function of $x=Q^{2} / m_{N}^{2}$ with $Q$ the photon's momentum and $m_{N}$ the nucleon's mass. Solid (black) curve, QCD-kindred prediction; dot-dashed (red) curve, contact-interaction result; dotted (green) curve, inferred meson-cloud contribution; and dashed (blue) curve, anticipated complete result. Right panel - Pauli transition form factor, $F_{2}^{*}(x)$, with same legend. Data in both panels: circles (blue) [20]; triangle (gold) [21]; squares (purple) [22]; and star (green) [23].

performed in Ref. [11] the first continuum treatment of this problem using the power of relativistic quantum field theory. It is such calculation which has allowed us to state that the Roper resonance appears to be the nucleon's first radial excitation whose unexpectedly low mass arise from a dressedquark core that is shielded by a meson-cloud.

The left panel of Fig. 4 depicts the Dirac transition form factor, $F_{1}^{*}$, which vanishes at $x=0$ owing to orthogonality between the proton and its radial excitation. Our calculation, solid (black) curve, agrees quantitatively in magnitude and qualitatively in trend with the data on $x \gtrsim 2$. The nature of our prediction owes fundamentally to the QCD-derived momentum-dependence of the propagators and vertices employed in formulating the bound-state and scattering problems. This point is further highlighted by the contact-interaction result (dot-dashed-red): with momentum-independent propagators and vertices, the prediction disagrees both quantitatively and qualitatively with the data. Experiment is evidently a sensitive tool with which to chart the nature of the quark-quark interaction and hence discriminate between competing theoretical hypotheses; and it is plainly settling upon an interaction that produces the momentum-dependent dressed-quark mass which characterises QCD [25-27].

The mismatch between our prediction and the data on $x \lesssim 2$ is also revealing. As seen previously, e.g. Refs. [10, 28, 29], this is the domain upon which meson-cloud contributions are expected to be important. An estimate of that contribution is provided by the dotted (green) curve in the left panel of Fig. 4. If this curve is added to our prediction, then one obtains the dashed (blue) curve, which is a least-squares fit to the data on $x \in[0,5]$. The correction curve has fallen to just $20 \%$ of its maximum value by $x=2$ and vanishes rapidly thereafter so that our prediction alone remains as the explanation of the data.

The right panel of Fig. 4 depicts the Pauli transition form factor, $F_{2}^{*}$. All observations made regarding $F_{1}^{*}$ also apply here, including those concerning the estimated meson-cloud contributions. Importantly, the existence of a zero in $F_{2}^{*}$, a prominent feature of the data, is not influenced by meson-cloud effects, although its precise location is. Thus any realistic approach to the proton-Roper transition must describe a zero in $F_{2}^{*}$. It is worth noting in addition that our prediction $F_{2}^{*}(x=0)=-0.65$, i.e. for the Pauli form factor at the photoproduction point, is consistent with contemporary experiment: $-0.58 \pm 0.02$ [21] and $-0.62 \pm 0.04$ [23] 
In two separate ways, the electromagnetically induced $N \rightarrow R$ transition can be considered as a sum of three distinct terms [10]:

- $\mathbf{T 1}=$ diquark dissection. $T 1 A-$ scalar diquark in both the initial- and final-state baryon, $T 1 B-$ pseudovector diquark in both the initial- and final-state baryon, and $T 1 C$ - a different diquark in the initial- and final-state baryon.

- $\mathbf{T 2}=$ scatterer dissection. $T 2 A$ - photon strikes a bystander dressed-quark, $T 2 B$ - photon interacts with a diquark, elastically or causing a transition scalar $\leftrightarrow$ pseudovector, and $T 2 C$ - photon strikes a dressed-quark in-flight, as one diquark breaks up and another is formed, or appears in one of the two associated "seagull" terms.

The anatomy of the $\gamma p \rightarrow R^{+}$Dirac and Pauli transition form factors is revealed when combining the information provided by the T1 and T2 dissections, see Fig. 5. The Dirac form factor is primarily given by the contribution in which a photon strikes a bystander dressed quark that is partnered by a scalar-diquark: $[u d]$. However, all other processes have non-negligible contributions. In exhibiting these features, $F_{1, p}^{*}$ shows marked qualitative similarities to the proton's elastic Dirac form factor ( $c f$. Fig. 3 in Ref. [28]).

In the case of the Pauli transition form factor, a single contribution is overwhelmingly important, viz. photon strikes a bystander dressed-quark in association with $[u d]$ in the proton and $R^{+}$. No other diagram makes a significant contribution. A comparison with Fig. 4 in Ref. [28] reveals that the same may be said for the dressed-quark core component of the proton's elastic Pauli form factor.

Given that the diquark content of the proton and $R^{+}$are almost identical, with the $\psi_{0} \sim u[u d]$ component contributing roughly $60 \%$ of the charge of both systems, the qualitative similarity between the proton elastic and proton-Roper transition form factors is not surprising. This observation immediately raises the issue of whether and how that similarity is transmitted into the flavour separated form factors.

If one supposes that $s$-quark contributions to the nucleon-Roper transitions are negligible, as is the case for nucleon elastic form factors, and assumes isospin symmetry, then a flavour separation of the transition form factors is accomplished by combining results for the $\gamma^{*} p \rightarrow R^{+}$and $\gamma^{*} n \rightarrow R^{0}$ transitions:

$$
F_{1(2), u}^{*}=2 F_{1(2)}^{*, p}+F_{1(2)}^{*, n}, \quad F_{1(2), d}^{*}=2 F_{1(2)}^{*, n}+F_{1(2)}^{*, p},
$$

where $p$ and $n$ are superscripts that indicate, respectively, the charged and neutral nucleon-Roper reactions. Our conventions are that $F_{1(2), u}^{*}$ and $F_{1(2), d}^{*}$ refer to the $u$ - and $d$-quark contributions to the equivalent Dirac (Pauli) form factors of the $\gamma^{*} p \rightarrow R^{+}$reaction, and the results are normalised such that the elastic Dirac form factors of the proton and charged-Roper yield $F_{1 u}\left(Q^{2}=0\right)=2$, $F_{1 d}\left(Q^{2}=0\right)=1$, thereby ensuring that these functions count $u$ - and $d$-quark content in the boundstates.

The upper panels of Fig. 6, depicting the flavour-separated Dirac transition form factor, show an obvious similarity to the analogous form factor determined in elastic scattering: the $d$-quark contribution is less-than half the $u$-quark contribution for momenta sufficiently far outside the neighbourhood of $x=Q^{2} / m_{N}^{2}=0$ within which they both vanish; and the $d$-quark contribution falls more rapidly after their almost coincident maxima. The noticeable difference, however, is the absence of a zero in $F_{1, d}^{*}$, which is a salient feature of the analogous proton elastic form factor.

The lower panels of Fig. 6 depict the flavour-separated Pauli transition form factor. In this instance the similarities are less obvious, but they are revealed once one recognises that the rescaling factors satisfy $\left|\kappa_{d}^{*} / \kappa_{u}^{*}\right|<\frac{1}{6} c f$. a value of $\sim \frac{2}{5}$ in the elastic case [28]. Accounting for this, the behaviour of the $u$ - and $d$-quark contributions to the charged-Roper Pauli transition form factor are comparable with 

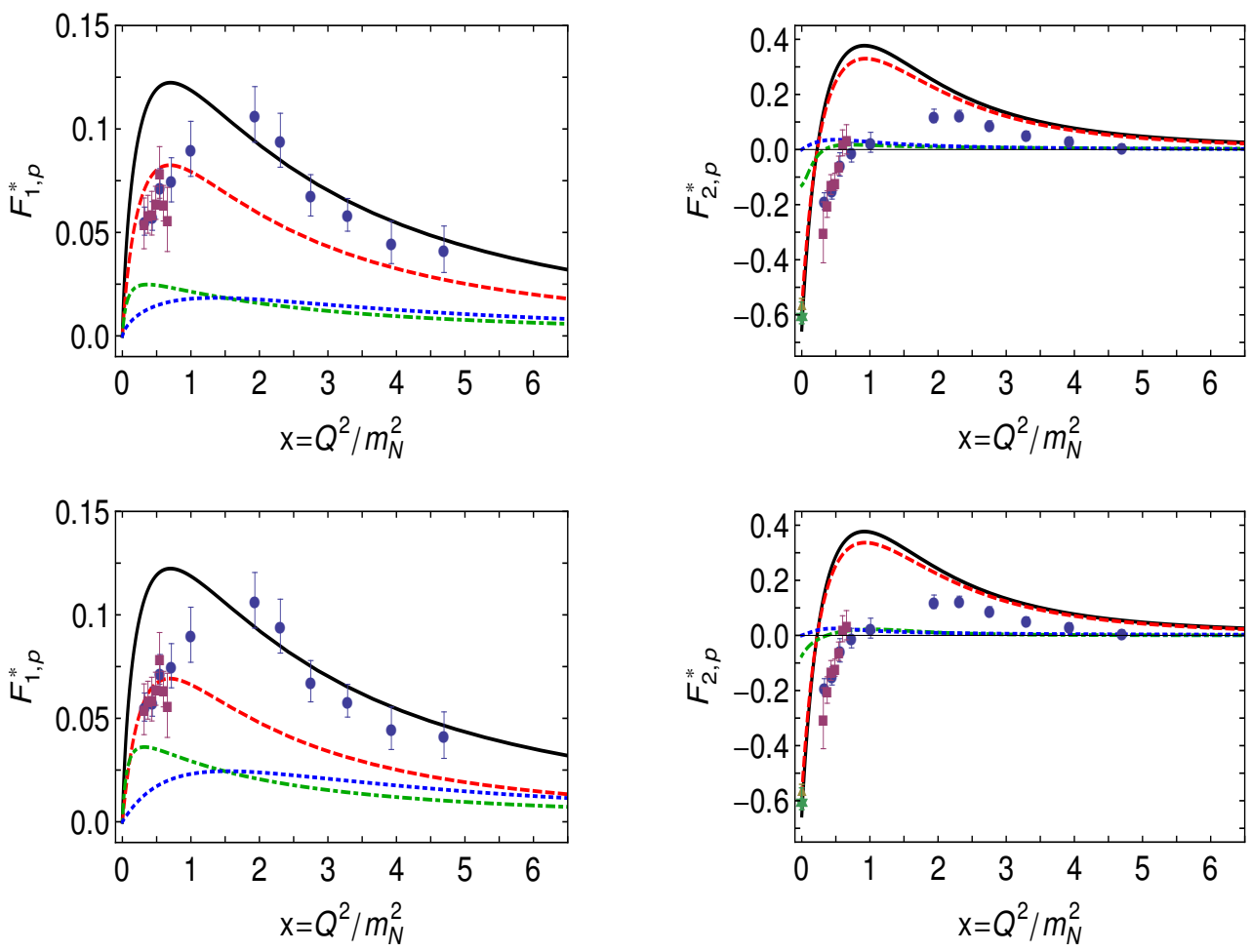

Figure 5. Left panels (Right panels) - The $\gamma^{*} p \rightarrow R^{+}$Dirac (Pauli) transition form factor, $F_{1, p}^{*}\left(F_{2, p}^{*}\right)$, as a function of $x=Q^{2} / m_{N}^{2}$, computed as described in Ref. [11] (solid black curve). Upper panels - diquark breakdown: T1A (dashed red), scalar diquark in both $p, R^{+} ; T 1 B$ (dot-dashed green), pseudovector diquark in both $p, R^{+}$; TIC (dotted blue), scalar diquark in $p$, pseudovector diquark in $R^{+}$, and vice versa. Lower panels - scatterer breakdown: $T 2 A$ (red dashed), photon strikes an uncorrelated dressed quark; $T 2 B$ (dot-dashed green), photon strikes a diquark; and $T 2 C$ (dotted blue), diquark breakup contributions, including photon striking exchanged dressed-quark. Data: circles (blue) [20]; squares (purple) [22]; triangle (gold) [21]; and star (green) [23].

the kindred contributions to the elastic form factor, especially insofar as the $d$-quark contribution falls dramatically on $x \gtrsim 4$ whereas the $u$-quark contribution evolves more slowly.

An explanation for the pattern of behaviour in Fig. 6 is much the same as that for the analogous proton elastic form factors because the diquark content of the proton and its first radial excitation are almost identical. In both systems, the dominant piece of the associated Faddeev wave functions is $\psi_{0}$, namely a $u$-quark in tandem with a $[u d]$ (scalar diquark) correlation, which produces $62 \%$ of each bound-state's normalisation [11]. If $\psi_{0}$ were the sole component in both the proton and chargedRoper, then $\gamma-d$-quark interactions would receive a $1 / x$ suppression on $x>1$, because the $d$-quark is sequestered in a soft correlation, whereas a spectator $u$-quark is always available to participate in a hard interaction. At large- $x$, therefore, scalar diquark dominance leads one to expect $F_{d}^{*} \sim F_{u}^{*} / x$. Naturally, precise details of this $x$-dependence are influenced by the presence of pseudovector diquark correlations in the initial and final states, which guarantees that the singly-represented quark, too, can participate in a hard scattering event, but to a lesser extent. 

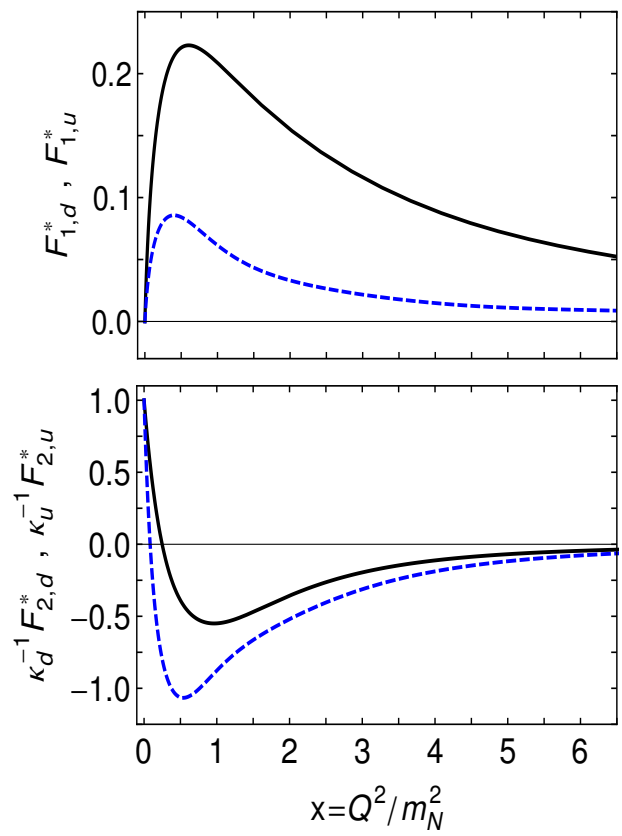
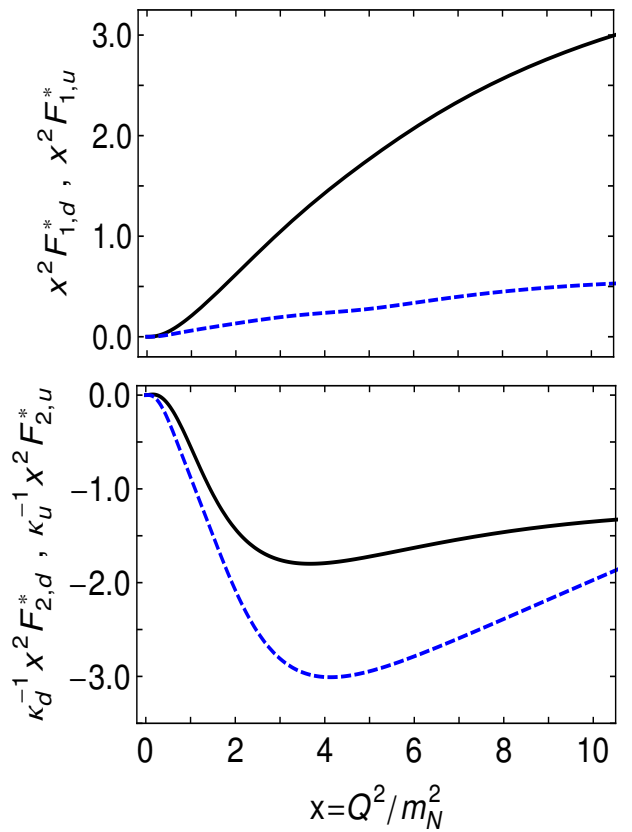

Figure 6. Left panels - Flavour separation $\gamma^{*} p \rightarrow R^{+}$transition form factors: $u$-quark, solid black; and $d$-quark, dashed blue. Upper-left panel - Dirac transition form factor. Lower-left panel - Pauli transition form factor, with $\kappa_{u}^{*}=F_{2, u}^{*}(0)=-0.91, \kappa_{d}^{*}=F_{2, d}^{*}(0)=0.14$. Right panels $-x^{2}=\left(Q^{2} / m_{N}^{2}\right)^{2}$-weighted behaviour of the flavour separated $\gamma^{*} p \rightarrow R^{+}$transition form factors: $u$-quark, solid black; and $d$-quark, dashed blue. Upper-right (lower-right) panel - Dirac (Pauli) transition form factor.

The infrared behaviour of the flavour-separated $\gamma^{*} p \rightarrow R^{+}$transition form factors owes to a complicated interference between the influences of orthogonality, which forces $F_{1, u}^{*}(0)=0=F_{1, d}^{*}(0)$, and quark-core and MB FSI contributions. However, whilst the latter pair act in similar ways for both elastic and transition form factors, orthogonality is a fundamental difference between the two processes and it is therefore likely to be the dominant effect at infrared momenta.

The information contained in Figs. 5 and 6 provides clear evidence in support of the notion that many features in the measured behaviour of $\gamma^{*} N \rightarrow R$ electromagnetic transition form factors are primarily driven by the presence of strong diquark correlations in the nucleon and its first radial excitation. In our view, inclusion of a "meson cloud" cannot qualitatively affect the salient features of these transition form factors, any more than it does the analogous nucleon elastic form factors [14, 30].

\section{Conclusions}

We have explained how the emergent phenomenon of dynamical chiral symmetry breaking ensures that Poincaré covariant analyses of the three valence-quark scattering problem in continuum quantum field theory yield a picture in which binding arises primarily through the sum of two separate contributions. One involves aspects of the non-Abelian character of QCD that are expressed in the strong running coupling and generate tight, dynamical colour-antitriplet quark-quark correlations. This attraction is magnified by quark exchange associated with diquark breakup and reformation, which 
is required in order to ensure that each valence-quark participates in all diquark correlations to the complete extent allowed by its quantum numbers.

The existence of strong diquark correlations inside the nucleon and its resonances has numerous observable consequences and we have presented herein some of them related with the nucleon's elastic and nucleon-to-Roper transition electromagnetic form factors.

In the case of the nucleon, we have seen that Poincare covariance, which demands the presence of dressed-quark orbital angular momentum in the nucleon, and dominant scalar diquark correlations are sufficient to understand empirical extractions of the flavour-separated Dirac and Pauli form factors, and the $Q^{2}$-behaviour of the proton's electromagnetic ratios.

The anatomy of the $\gamma^{*} p \rightarrow R^{+}$Dirac and Pauli transition form factors has been presented. We concluded that the contribution in which the photon strikes a bystander $u$-quark in association with a scalar diquark $[u d]$ is dominant for both Dirac and Pauli transition form factors. Moreover, their flavour separated versions show similarities to the analogous form factors determined in elastic scattering. In the case of Dirac transition form factor, the $d$-quark contribution is less-than half the $u$-quark contribution for momenta sufficiently far outside the neighbourhood of $Q^{2}=0$; and the $d$-quark contribution falls more rapidly at very large momenta. The similarities are less obvious in the case of the Pauli transition form factor but one can appreciate that the $d$-quark contribution falls dramatically at large momenta whereas the $u$-quark contribution evolves more slowly.

\section{Acknowledgements}

The material described in this contribution is drawn from work completed in collaboration with numerous excellent people, to all of whom we are greatly indebted. We thank R. Gothe, V. Mokeev and V. Burkert for suggesting this problem, and T.S.-H. Lee and T. Sato for numerous informative discussions. This work was supported by: the Alexander von Humboldt Foundation; and the U.S. Department of Energy, Office of Science, Office of Nuclear Physics, under contract no. DE-AC02-06CH11357.

\section{References}

[1] I.C. Cloët, C.D. Roberts, Prog. Part. Nucl. Phys. 77, 1 (2014)

[2] J. Segovia, C.D. Roberts, S.M. Schmidt, Phys. Lett. B750, 100 (2015)

[3] C. Alexandrou, P. de Forcrand, B. Lucini, Phys. Rev. Lett. 97, 222002 (2006)

[4] R. Babich, N. Garron, C. Hoelbling, J. Howard, L. Lellouch, C. Rebbi, Phys. Rev. D76, 074021 (2007)

[5] G. Eichmann, H. Sanchis-Alepuz, R. Williams, R. Alkofer, C.S. Fischer, Prog. Part. Nucl. Phys. 91, 1 (2016)

[6] G. Eichmann, C.S. Fischer, H. Sanchis-Alepuz (2016), arXiv : hep-ph/1607. 05748

[7] J. Segovia, C.D. Roberts, Phys. Rev. C94, 042201 (2016)

[8] J. Segovia, C. Chen, C.D. Roberts, S. Wan, Phys. Rev. C88, 032201 (2013)

[9] J. Segovia, C. Chen, I.C. Cloët, C.D. Roberts, S.M. Schmidt, S. Wan, Few Body Syst. 55, 1 (2014)

[10] J. Segovia, I.C. Cloët, C.D. Roberts, S.M. Schmidt, Few Body Syst. 55, 1185 (2014)

[11] J. Segovia, B. El-Bennich, E. Rojas, I.C. Cloët, C.D. Roberts, S.S. Xu, H.S. Zong, Phys. Rev. Lett. 115, 171801 (2015)

[12] G. Eichmann, R. Alkofer, A. Krassnigg, D. Nicmorus, Phys. Rev. Lett. 104, 201601 (2010)

[13] D.J. Wilson, I.C. Cloet, L. Chang, C.D. Roberts, Phys. Rev. C85, 025205 (2012) 
[14] I.C. Cloët, W. Bentz, A.W. Thomas, Phys. Rev. C90, 045202 (2014)

[15] G.D. Cates, C.W. de Jager, S. Riordan, B. Wojtsekhowski, Phys. Rev. Lett. 106, 252003 (2011)

[16] O. Gayou et al., Phys. Rev. C64, 038202 (2001)

[17] V. Punjabi et al., Phys. Rev. C71, 055202 (2005), [Erratum: Phys. Rev. C71, 069902(2005)]

[18] A.J.R. Puckett et al., Phys. Rev. Lett. 104, 242301 (2010)

[19] A.J.R. Puckett et al., Phys. Rev. C85, 045203 (2012)

[20] I.G. Aznauryan et al. (CLAS), Phys. Rev. C80, 055203 (2009)

[21] M. Dugger et al. (CLAS), Phys. Rev. C79, 065206 (2009)

[22] V.I. Mokeev et al. (CLAS), Phys. Rev. C86, 035203 (2012)

[23] K.A. Olive et al. (Particle Data Group), Chin. Phys. C38, 090001 (2014)

[24] I.G. Aznauryan, V.D. Burkert, Prog. Part. Nucl. Phys. 67, 1 (2012)

[25] P.O. Bowman, U.M. Heller, D.B. Leinweber, M.B. Parappilly, A.G. Williams, J.b. Zhang, Phys. Rev. D71, 054507 (2005)

[26] M.S. Bhagwat, P.C. Tandy, AIP Conf. Proc. 842, 225 (2006)

[27] C.D. Roberts, Prog. Part. Nucl. Phys. 61, 50 (2008)

[28] I.C. Cloët, G. Eichmann, B. El-Bennich, T. Klahn, C.D. Roberts, Few Body Syst. 46, 1 (2009)

[29] C.D. Roberts, J. Phys. Conf. Ser. 630, 012051 (2015)

[30] I.C. Cloët, G.A. Miller, Phys. Rev. C86, 015208 (2012) 